\title{
Costly Outcomes of Education-Another Case of Nature Versus Nurture
}

$\mathbf{T}$ he costs associated with residency training can be tracked to several sources, including the additional costs of patient care in teaching settings. While some costs of residency training are fixed and are inherent to the training program itself, other costs, such as those associated with patient care in settings that include teaching, may be more amenable to change. This issue is significant when funding for graduate medical education is threatened and academic medical centers find themselves competing with centers that avoid teaching "at all costs."

Medical care that involves residents probably costs more than other medical care, and necessarily so. But might some of that cost yield to educational programs that focus specifically on cost containment? That depends on whether residents practice costly medicine because of nature or nurture, specifically whether the increased patientcare costs associated with residency training are attributable to the residents themselves or to the environment in which the residents are trained. We believe that residents assume standards of practice from the environments in which they learn. They do not bring with them a fixed notion of best practice; rather, they develop that notion from what they see and what they are taught. Residents create what they observe. If triangles had a god, Montesquieu said, he would have three sides.

Many of us can remember a different time. Call it the era of cost-plus medicine or simply "before managed care." It was an era when every patient with a new diagnosis of cirrhosis was tested for antismooth muscle antibodies to find autoimmune hepatitis, and every patient with COPD had to have an alpha-one antitrypsin level before the attending arrived. Tests took on a whole new meaning: they demonstrated our sophistication with differential diagnosis, and they indicated the extent of our knowledge. Things certainly have changed.

It is important to know whether these changes have affected patient outcomes and the cost of patient care. In this issue of JGIM, Griffith and colleagues review studies that measure the impact of residency programs on patient outcomes and resource utilization. ${ }^{1}$ Morbidity and mortality appear not to be affected by the presence of residency programs, nor by the residents' level of experience, at least not in systems that couple inexperienced residents with more experienced residents and teaching faculty. It seems, however, that residency programs do, or did, have a decided effect on costs. Residents in many studies were not cost conscious, as reflected by the number of tests and ancillary studies they ordered.

Whether that's still the case cannot be determined from this review. As the authors point out, most of the studies on patient costs associated with residency training were done in the 1970 s and 80 s. Some recent data ${ }^{2}$ and our personal experience suggest that residency train- ing today may not be as strongly associated with increased patient costs and utilization. In fact, Griffith cites one study ${ }^{3}$ that found a decreased length of stay when the teaching service of one hospital was compared with the nonteaching service. The question of whether this is a general or an isolated phenomenon awaits further study. The issue is important and should be studied further by those interested in research on medical education. But absent such data, we believe several suggestions can be made.

We believe that residents trained in cost-conscious settings will be cost conscious. The salient question of Griffith's paper is not, "Do residents inherently drive costs up?" but rather, "What must the training environment provide to keep those costs down?" Obviously, residents need to understand prospective payment and managed care. They need to develop the clinical judgment that allows skilled physicians to consider cost without lowering the quality of care. They need to learn to practice within critical pathways and disease-management protocols, in part by helping develop these protocols

Griffith reviewed studies suggesting that during the 1980s residents were not sensitive to the cost of medications. Who was? Today, residents need to learn how pharmacy costs affect institutional costs and how drug costs affect patients directly. Pharmacists rounding with residents can stimulate discussions regarding medication costs, and restrictions on unnecessary prescribing habits should be part of every residency program. It's just a fact of life.

Also, Griffith found that increased length of stay was associated with residency training in some studies, although in one study the trend went the other way. ${ }^{3}$ Today, residents can learn to care for patients more efficiently if the training environment encourages this. One component of such an environment is coordinated communication around discharge planning. Another is an integrated team approach, including social workers, therapists, and nurses-another fact of life.

Graduate medical education is not cost-free. There are fixed costs associated with residency training that cannot be eliminated. The training process requires that residents participate meaningfully in realistic settings. It requires that residents be allowed to assess patients independently, develop differential diagnoses, and initiate evaluations. Training implies that the clinical acumen of residents will not be as sharp as that of their faculty supervisors. Residents may take longer and require more tests and procedures to arrive at the same conclusion as a senior faculty member. For faculty, the very presence of residents means time away from patient care and funded research. But no one is talking about abandoning medicine's teaching mission, just changing the environment in which that teaching takes place. The new environment must weave systems for cost containment into the systems for education. Un- 
necessary patient costs associated with resident education need to be eliminated, along with the incentives that encouraged ruling out obscure diagnoses and ordering excessive tests.

Medical centers committed to teaching must work the system at both ends. Both mission and margin must be pursued. In these hard times-and there is no reason to believe they are getting any easier-the costs associated with residency training must be controlled. A commitment to medical education is a commitment to integrating the concepts of cost-effectiveness into residency training programs. We believe residents can learn to practice costeffective medicine, and the fact that they may not have done so in the past is more of a challenge than a fact of
life.-LisA Bellini, MD and JACK ENDE, MD, Hospital of the University of Pennsylvania and the University of Pennsylvania School of Medicine, Philadelphia.

\section{REFERENCES}

1. Griffith $\mathrm{CH}$, Rich EC, Hillson SD, Wilson JF. Internal medicine residency training and outcomes. J Gen Intern Med. 1997;12:390-6.

2. Haywood RA, Manning WG, McMahon LF, Bernard AM. Do attending or resident physician practice styles account for variations in hospital resources use? Med Care. 1994;32:788-94.

3. Udvarhelyi IS, Rosborough T, Lofgren RP, Lurie N, Epstein AM. Teaching status and resource use for patients with mycardial infarction: a new look at the indirect costs of graduate medical education. Am J Public Health. 1990;80:1095-100.

\section{REFLECTIONS \\ Degrees of Death (observations on surgery)}

they say he's dying by degrees and cut his legs off at the knees to wipe away the dark disease his feet fell from his trunk. a rumpled naked man from whom came blood, a pooling liquidbloom to grow inside the sterile room plucked up by netted gauze.

what wisdom can we reap from pain? what process do we try to name? the man was healed to make him lame and lay upon the table. we all are dying by degrees and pay for time in paper fees we still have feet beneath our knees and only need reminding.

there may be pain beneath the skin the buried stone diseased within but before you push the needle in consider first the patient.

George Veech Charlottesville, Va. Finalist 1997 Creative Medical Writing Contest 